\title{
Butyrylated starch is less susceptible to enzymic hydrolysis and increases large-bowel butyrate more than high-amylose maize starch in the rat
}

\author{
Balázs H. Bajka ${ }^{1,2,3}$, David L. Topping ${ }^{1,3 *}{\text { Lynne } \text { Cobiac }^{3} \text { and Julie M. Clarke }}^{1,3}$ \\ ${ }^{1}$ CSIRO Human Nutrition, Kintore Avenue, PO Box 10041, Adelaide BC 5000, Australia \\ ${ }^{2}$ Discipline of Physiology, School of Molecular and Biomedical Sciences, The University of Adelaide 5005, Adelaide, Australia \\ ${ }^{3}$ Preventative Health National Research Flagship, Kintore Avenue, PO Box 10041, Adelaide BC 5000, Australia
}

(Received 11 November 2005 - Revised 10 March 2006 - Accepted 16 March 2006)

\begin{abstract}
Large-bowel fermentation of resistant starch produces SCFA that are believed to be important in maintaining visceral function. High-amylose maize starch (HAMS) and acylated starches are sources of resistant starch and are an effective means of increasing colonic SCFA. Cooking increases digestibility of starches but its effects on the capacity of these starches to raise large-bowel SCFA are unknown. We have examined the effects of cooking of HAMS and butyrylated HAMS (HAMSB) on amylolysis in vitro and their capacity to raise caeco-colonic SCFA in rats. The starches were boiled in excess water and microwaved, followed by drying at $100^{\circ} \mathrm{C}$. Cooking increased in vitro glucose release for both starches but significantly less from HAMSB. Rat growth rates were unaffected when fed cooked resistant starch. Digesta $\mathrm{pH}$ was increased in the caecum and proximal colon of rats fed cooked HAMS. Distal colonic $\mathrm{pH}$ was highest in rats fed cooked HAMSB. Factorial analyses $(2 \times 2)$ of caecal SCFA pools showed significant differences between HAMS and HAMSB, and that cooking significantly lowered caecal butyrate pools. Portal venous butyrate concentrations were higher in both HAMSB groups than those fed HAMS. The data suggest that HAMSB is less susceptible to in vitro amylolysis than HAMS following cooking and delivers more butyrate to rat caecum than HAMS. This attribute may be useful in food applications for specific delivery of SCFA to the colon. Preparation of carbohydrates to simulate human food in animal experiments may be important to assess nutritional and physiological effects accurately.
\end{abstract}

Resistant starch: Processing: Short-chain fatty acids: Large bowel

Increased luminal SCFA are believed to improve large-bowel health through a variety of mechanisms. These include increasing colonic blood flow (Topping \& Clifton, 2001), improving mineral and water absorption and the maintenance of low luminal $\mathrm{pH}$, particularly in the proximal colon, which may be beneficial in the prevention and overgrowth of $\mathrm{pH}$-sensitive pathogenic bacteria (Kleessen et al. 1997). Butyrate, in particular, has been implicated in specific functions involving the epithelial metabolism (Ahmad et al. 2000) and cell cycling (Dehghan-Kooshkghazi \& Mathers, 2004; Scheppach \& Weiler, 2004), immune response (Andoh et al. 2003; Inatomi et al. 2005) and intestinal motility (Rondeau et al. 2003).

The importance of carbohydrate fermentation in increasing large-bowel SCFA has been established (Topping et al. 1997; Young et al. 2005). It has been suggested that SCFA produced from fermentable dietary carbohydrates are higher in populations with low risk of serious bowel disease (Ahmed et al. 2000). It appears that one of the major sources of dietary carbohydrate available for fermentation is resistant starch (RS) (Andoh et al. 2003; Topping et al. 2003). RS is defined as 'the sum of starch and products of starch degradation not absorbed in the small intestine of healthy individuals' (Asp,
1992). RS is an important dietary component and is found in a number of foods, but it appears that intakes are low in affluent Westernised countries at risk of serious large-bowel disease (Champ et al. 2003). The RS content of foods may be increased through the addition of specific ingredients such as high-amylose starches (Brown et al. 1995). However, dietary studies with such products in animals have used ungelatinised, i.e. raw, products which are not truly representative of human foods. Maize-starch mixtures that have been processed by extrusion display different physico-chemical properties such as increased water solubility, decreased gel viscosity and a greater percentage of smaller-molecularweight oligosaccharides (Ozcan \& Jackson, 2005). This could result in increased small-intestinal amylolysis, and is supported by in vitro studies that demonstrated a significant change in the structure of chestnut flour following roasting and a corresponding increased in vitro amylolysis (Pizzoferrato et al. 1999). Processing has been found to increase in vitro amylolysis in rice, pearl barley and foxtailed millet compared with the raw cereals (Lee \& Chang, 2004). A recent study confirmed a $50 \%$ reduction in the fermentative capacity in vivo of a RS (80\% amylose) in volunteers 
fed pancakes containing the high-amylose starch, compared with the raw starch dispersed in a cold beverage (Symonds et al. 2004).

We have shown that starches acylated with acetate, propionate or butyrate are effective in raising total SCFA concentrations and pools throughout the large bowel with the greatest increase being in the acid which had been esterified (Annison et al. 2003). It has been shown in rats that these starches survived small-intestinal amylolysis and entered the large bowel where the SCFA was liberated by bacterial esterases (Morita et al. 2005). While acylated starches offer the potential for specific delivery of SCFA to the large bowel, these studies were carried out with ungelatinised (i.e. raw) starches. Although it is likely that the ester bond would be stable to moist heat, it remained necessary to ascertain whether cooking will have any effect on their SCFA delivery capability.

In the present studies we investigated the effects of feeding high-amylose maize starch (HAMS) and butyrylated HAMS (HAMSB) on caeco-colonic SCFA pools in rats. We have also examined the effect of cooking on the resistance of starches to amylolysis in vitro and SCFA pools in vivo.

\section{Materials and methods}

Animals

Adult male Sprague-Dawley rats were sourced from the Animal Resource Centre, Murdoch University, Western Australia and allowed $7 \mathrm{~d}$ to stabilise before the experimental period. Animals were housed in wire-based cages in a room of controlled temperature $\left(22-24^{\circ} \mathrm{C}\right)$ and lighting (lights on between 08.30 and 19.30 hours). The rats were allowed free access to a standard pelleted diet during the adaptation period (Ridley Agriproducts, Murray Bridge, SA, Australia). The experiment was approved by the animal ethics committees of Commonwealth Scientific and Industrial Research Organisation Human Nutrition (CSIRO) and the University of Adelaide (Australia), and complied with the
Australian code of practice for the care and use of animals for scientific purposes (National Health and Medical Research Council, 2004).

\section{Experimental diets}

HAMS (Hylon VII) and HAMSB were obtained from National Starch and Chemical Company (Bridgewater, NJ, USA). The degree of substitution of butyrate in HAMSB was 0.23 indicating one esterified butyryl side chain approximately every four glucosyl units of starch. The compositions of the test diets are listed in Table 1.

HAMS and HAMSB were cooked in water $(100 \mathrm{~g}$ starch per $200 \mathrm{ml}$ water). Cooking was initially on a hotplate with continuous mixing until the material thickened, followed by microwaving $(1100 \mathrm{~W}$ for $10 \mathrm{~min})$ to avoid burning until starch was gelatinised. Starches were transferred immediately to a drying oven and dried overnight at $100^{\circ} \mathrm{C}$ to minimise retrogradation and the reformation of RS crystals (Brown et al. 2003). Starches were removed periodically and blended in a food processor to aid in drying. The final products were fine powders.

\section{In vitro analysis of starches}

The susceptibility of the starches to amylolysis was measured in vitro to determine the likely resistance of the starches in vivo. Raw and cooked standard maize starch was included as a positive control. Each starch $(100 \mathrm{mg})$ was dispersed in $0.2 \mathrm{M}$-acetate buffer (6 ml; $\mathrm{pH} \mathrm{6.0)}$, containing $10 \mathrm{mg} \alpha$-amylase (speedase PNA8; Halcylon Proteins Pty Ltd, Australia) and $0.86 \mathrm{mg}$ amyloglucosidase (Sigma Chemical Co., St Louis, MO, USA) and incubated in a shaking incubator at $37^{\circ} \mathrm{C}$ for $24 \mathrm{~h}$. Following incubation samples were centrifuged ( $50 \mathrm{~g}$ for $2 \mathrm{~min}$ ) and the supernatant fraction was assayed for glucose. Briefly a $10 \mu \mathrm{l}$ sample was pipetted in triplicate onto a ninety-six-well plate followed by $200 \mu \mathrm{l}$ glucose oxidase reagent (Thermo Electron Corporation, Victoria, Australia). The plate was incubated at room temperature for

Table 1. Composition of experimental diets ( $\mathrm{g} / \mathrm{kg}$ diet) based on the AIN-93G and containing $10 \%$ resistant starches*

\begin{tabular}{lcccc}
\hline & \multicolumn{3}{c}{ Diet } \\
\cline { 2 - 5 } Ingredient & HAMS & 'Cooked' HAMS & HAMSB & 'Cooked' HAMSB \\
\hline Casein & 200 & 200 & 200 & 200 \\
Standard maize starch & 430 & 430 & 430 & 430 \\
HAMS & 100 & - & - & - \\
'Cooked' HAMS & - & 100 & - & - \\
HAMSB & - & - & 100 & 100 \\
'Cooked' HAMSB & - & - & - & 100 \\
Sucrose & 100 & 100 & 100 & 70 \\
Sunflower-seed oil & 70 & 70 & 70 & 50 \\
Fibre ( $\alpha$-cellulose) & 50 & 50 & 35 & 10 \\
Mineral mix† & 35 & 35 & 10 & 3 \\
Vitamin mix† & 10 & 10 & 3 & 2.5 \\
L-Cystine & 3 & 3 & 2.5 & 0.014 \\
Choline bitartrate & 2.5 & 2.5 & 0.014 & \\
Tert-butylhydroquinone & 0.014 & 0.014 & \\
\hline HAMS, high-amylose maize starch; HAMSB, butyrylated high-amylose maize starch. & \\
*All diets were based on the AIN-93G formulation (Reeves et al. 1993). & & \\
†AIN-93G mineral and vitamin mixes were obtained pre-made from MP Biomedicals Inc. (Solon, OH, USA).
\end{tabular}


$20 \mathrm{~min}$ and read on a microplate reader at $505 \mathrm{~nm}$. Glucose concentrations were determined using a standard curve and percentage release by calculating the glucosyl units liberated from $100 \mathrm{mg}$ starch.

\section{Sample collection and analytical procedures}

Thirty-two rats (body weight 200-260 g) were divided into four equal groups and fed one of the four diets detailed in Table 1 for a period of $10 \mathrm{~d}$. Individual rat body weights and food intakes for each cage were measured daily. At the conclusion of the experimental period rats were exsanguinated under halothane anaesthesia. The lengths of the small and large intestine and caecal, colonic, liver and spleen weights were measured post mortem.

Separate digesta samples from the caecum, proximal and distal colon were collected for SCFA analysis and DM determinations. Briefly, digesta samples were weighed and diluted $1: 3$ in solvent containing $1.68 \mathrm{~mm}$-heptanoic acid (Sigma Chemical Co.) internal standard ( $\mathrm{pH} \mathrm{7.0),} \mathrm{homogenised,} \mathrm{pH}$ measured, then centrifuged at $2000 \mathrm{~g}$ for $15 \mathrm{~min}$. The supernatant fraction $(150 \mu \mathrm{l})$ was added to $30 \mu \mathrm{l} 10 \%$ phosphoric acid and purified by freeze transfer sublimation. The sublimate was analysed using GLC (6890N network GC system; Aligent Technologies Inc., Palo Alto, CA, USA); the column was FFAP $30 \mathrm{~m} \times 0.53 \mathrm{~mm}$ internal diameter (Phenomenex, Torrance, CA, USA). The solid fraction was then dried in an oven at $80^{\circ} \mathrm{C}$ for $24 \mathrm{~h}$ to determine the DM fraction of the digesta samples. DM samples were then hydrolysed to determine the amount of SCFA remaining esterified to starch in the digesta. DM digesta $(100 \mathrm{mg})$ was hydrolysed using $1.25 \mathrm{ml} 0.45 \mathrm{M}$-sodium hydroxide for $2 \mathrm{~h}$, diluted $1: 3$ (w/w) in solvent containing $100 \mathrm{~mm}$-2-ethylbutyric acid, then purified and analysed using GLC.

Portal vein plasma SCFA were determined by diethyl ether extraction as described previously (Murase et al. 1995).

\section{Statistical methods}

Statistical analysis was performed using GraphPad Prism version 4.0 computer software (GraphPad Software, San Diego, CA, USA). Where appropriate, the effects of starch type (HAMS and HAMSB) and processing (raw or cooked) and their interactions were evaluated by $2 \times 2$ factorial analysis using two-way ANOVA. One-way ANOVA using Tukey's post hoc test was used to determine differences among all test diets. All data are expressed as mean values with their standard errors, with statistical significance indicated when $P<0 \cdot 05$.

\section{Results}

In vitro hydrolysis

Only a small fraction of glucose $(6 \%)$ was released from uncooked HAMSB in vitro compared with uncooked HAMS, where $26 \%$ of glucose was liberated (Fig. 1). Cooking resulted in a very significant $(P<0.001)$ increase in glucose release from both starches $(75 \%$ for HAMS and $43 \%$ for HAMSB) but HAMSB remained more resistant to amylolysis

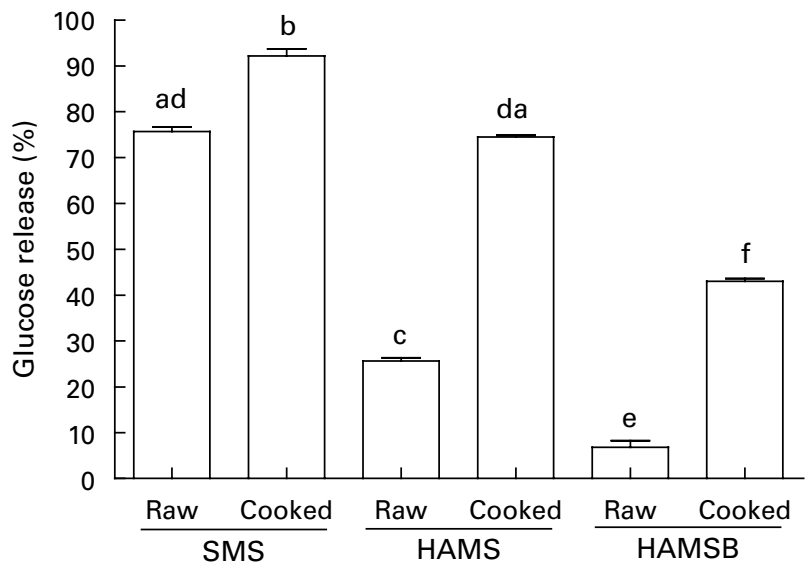

Fig. 1. The percentage glucose release from $100 \mathrm{mg}$ starch following amylolysis in vitro of raw and cooked standard maize starch (SMS), high-amylose maize starch (HAMS) and butyrylated HAMS (HAMSB). Each starch was assayed in triplicate and values are means, with their standard errors represented by vertical bars. ${ }^{a, b, c, d, e, f}$ Mean values with unlike letters were significantly different by one-way ANOVA $(P<0.001)$. Analysis by two-way ANOVA showed a significant effect of starch type and cooking $(P<0.0001)$.

than HAMS (Fig. 1). When standard maize starch was cooked, glucose release increased significantly from $76 \%$ to $92 \%$.

\section{Animal feeding trial}

There were no significant differences in food intakes or growth rates of rats fed any of the diets (data not shown). Caecal digesta wet weights were similar in rats fed both ungelatinised starches (Fig. 2). However, statistical analysis showed that there were significant effects of treatment on digesta weight. Cooking decreased $(P<0.02)$ and butyrylation increased $(P<0.05)$ digesta wet weight in the caecum. Neither starch type nor cooking significantly affected digesta wet weight in the proximal or distal colon.

In the caecum and proximal colon, digesta $\mathrm{pH}$ was lower $(P<0.01)$ in rats fed raw HAMS or raw or cooked HAMSB than in those fed cooked HAMS (Fig. 3). In contrast, distal

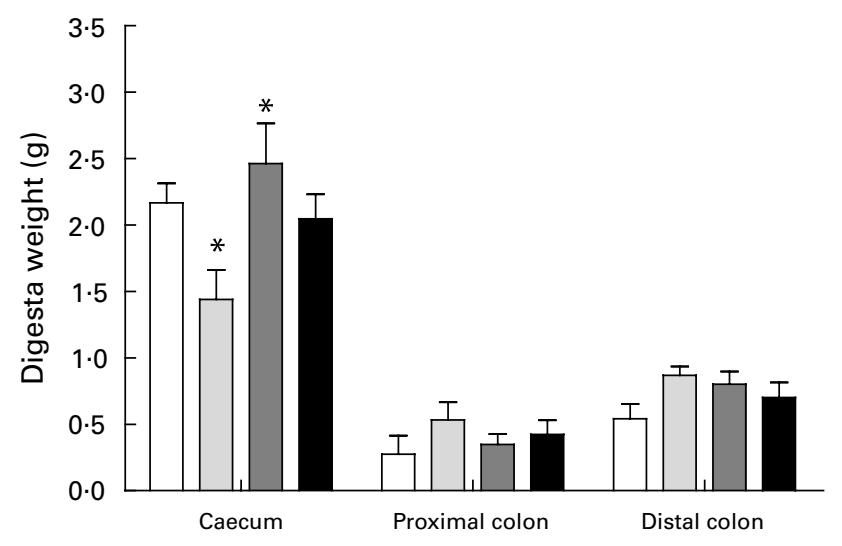

Fig. 2. Digesta weights from the caecum, proximal colon and distal colon from rats fed high-amylose maize starch (HAMS; $\square$ ), 'cooked' HAMS $(\square)$, butyrylated HAMS (HAMSB; $\square$ ) and 'cooked' HAMSB (ם). Data are means $(n 8)$ with their standard errors represented by vertical bars. ${ }^{*}$ Mean values were significantly different by one-way ANOVA $(P<0.05)$. A significant effect of cooking was observed in the caecum by two-way ANOVA $(P<0.02)$. 


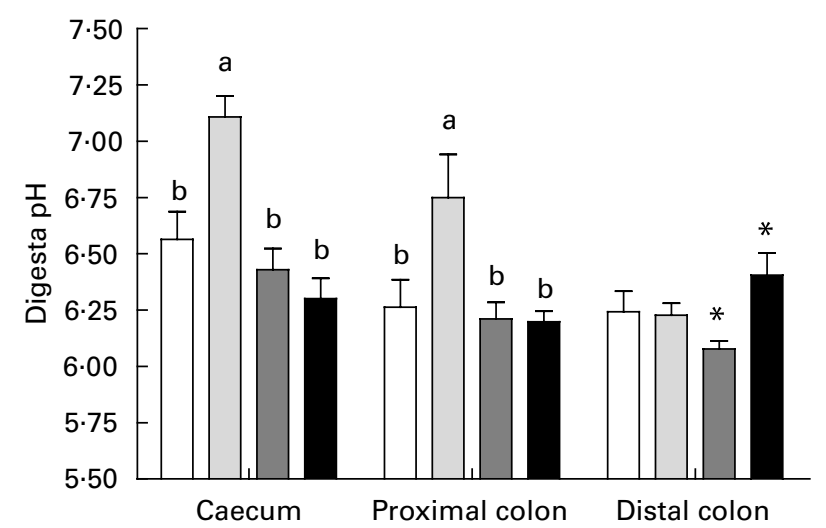

Fig. 3. Digesta $\mathrm{pH}$ from the caecum ( $n 8)$, proximal colon ( $n 4-7)$ and distal colon ( $n$ 7-8) from rats fed high-amylose maize starch (HAMS; $\square$ ), 'cooked' HAMS ( $\square$ ), butyrylated HAMS (HAMSB; $\square$ ) and 'cooked' HAMSB ( $\square$ ). Data are means with their standard errors represented by vertical bars. ${ }^{a, b}$ Within each caeco-colonic region, mean values with unlike letters were significantly different $(P<0.01)$. * Mean value was significantly different from that for HAMSB in the distal colon $(P<0.01)$. Overall, a two-way ANOVA demonstrated a significant difference due to cooking in the caecum and distal colon $(P<0.05)$.

colonic digesta $\mathrm{pH}$ was higher in rats fed cooked HAMSB compared with raw HAMSB $(P<0.05)$ although $\mathrm{pH}$ remained $<7 \cdot 0$ in all groups.

SCFA pools (Figs. 4, 5 and 6) were higher in the caecum than in either the proximal or distal colon. Two-way ANOVA showed that butyrylation of HAMS raised caecal acetate pools $(P<0.02)$ and that the decrease due to cooking was not significant $(P=0 \cdot 09)$. There were no significant differences in caecal acetate or propionate pools among the dietary groups; however, HAMSB (raw and cooked) had greater acetate pools than HAMS (raw and cooked). Caecal butyrate pools of the rats fed raw and cooked HAMSB were higher than the pools of both the raw and cooked HAMS groups $(P<0 \cdot 001)$. Cooking also reduced the butyrate pools regardless of starch type $(P<0 \cdot 05)$. No significant differences in SCFA pools

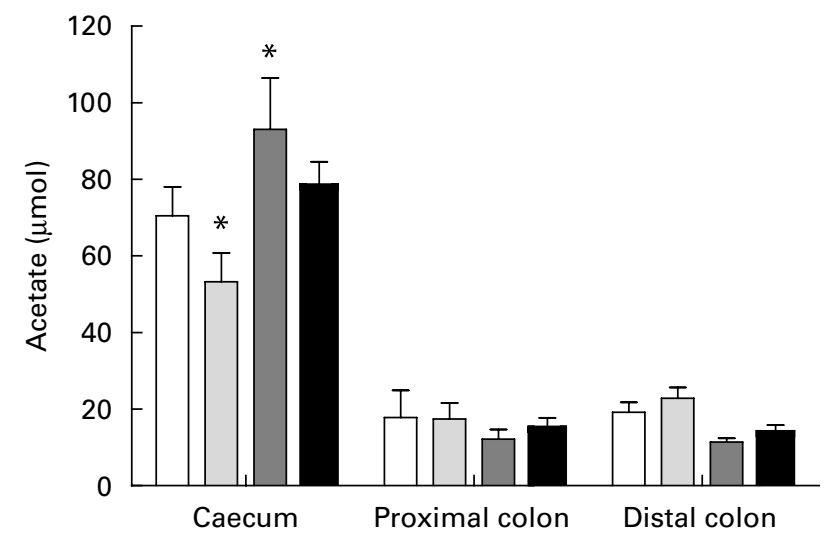

Fig. 4. Acetate pools in the liquid phase of the digesta from the caecum ( $n 8)$, proximal colon ( $n$ 4-7) and distal colon $(n 7-8)$ from rats fed high-amylose maize starch (HAMS; $\square$ ), 'cooked' HAMS $(\square)$, butyrylated HAMS (HAMSB; $\square)$ and 'cooked' HAMSB (ם). Data are means with their standard errors represented by vertical bars. * Mean values were significantly different by oneway ANOVA $(P<0.05)$. Significant differences in starch type were observed by two-way ANOVA in the caecum $(P<0.02)$ and distal colon $(P<0.001)$.

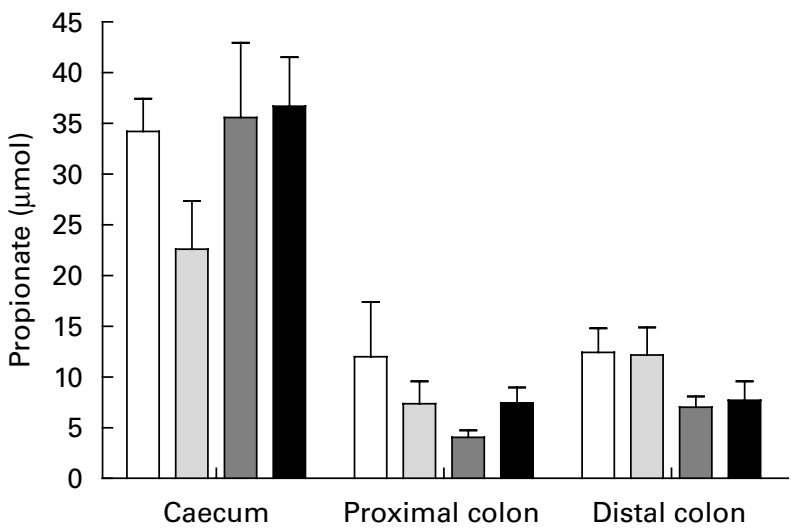

Fig. 5. Propionate pools in the liquid phase of the digesta from the caecum ( $n$ 8), proximal colon $(n 4-7)$ and distal colon $(n 7-8)$ from rats fed high-amylose maize starch (HAMS; $\square$ ), 'cooked' HAMS ( $\square$ ), butyrylated HAMS (HAMSB; $\square$ ) and 'cooked' HAMSB (ם). Data are means with their standard errors represented by vertical bars. Significant differences in starch type were observed by two-way ANOVA in the distal colon $(P=0.026)$.

were observed in the proximal colon among groups; however, in the distal colon both HAMS groups displayed more acetate $(P<0.001)$ and propionate $(P<0.05)$ but not butyrate. Although there were differences in percentage glucose release in vitro of raw and cooked HAMS (Fig. 1), there were no significant reductions due to cooking in total caeco-colonic SCFA (data not shown).

Alkaline hydrolysis of dried caeco-colonic digesta samples releases SCFA esterified to the starch molecules that can then be measured. The amount of butyrate released from $100 \mathrm{mg}$ digesta remained similar throughout the large bowel (Fig. 7). The amount of butyric side chains remaining esterified to the starch in the caeco-colonic digesta samples was significantly less than the estimated consumption over the previous $24 \mathrm{~h}$ period of the experiment as shown in Fig. 7 (diet butyrate pool $=$ butyrate liberated by alkaline hydrolysis $\times 24 \mathrm{~h}$ food intake).

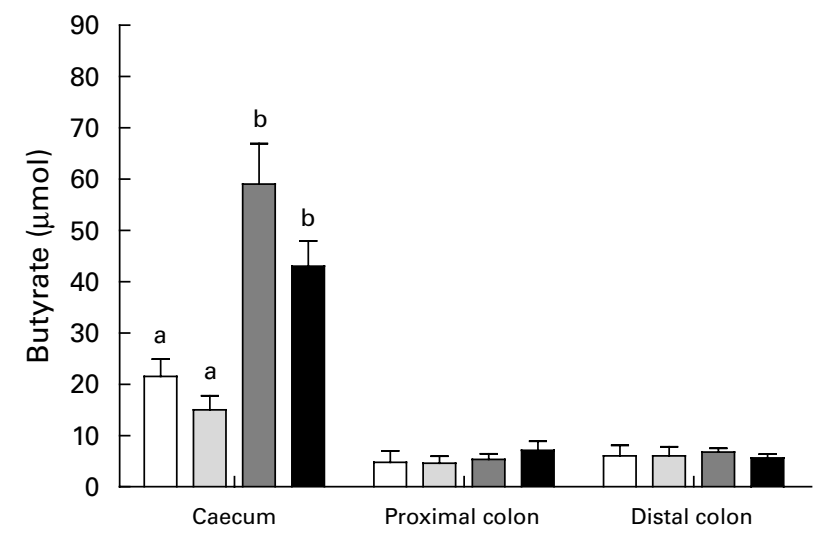

Fig. 6. Butyrate pools in the liquid phase of the digesta from the caecum $(n 8)$, proximal colon ( $n 4-7)$ and distal colon $(n 7-8)$ from rats fed high-amylose maize starch (HAMS; $\square$ ), 'cooked' HAMS ( $\square$ ), butyrylated HAMS (HAMSB; $\square$ ) and 'cooked' HAMSB (ם). Data are means with their standard errors represented by vertical bars. ${ }^{a, b}$ Mean values with unlike letters were significantly different by one-way ANOVA $(P<0.001)$. Significant differences in starch type $(P<0.0001)$ and cooking $(P<0.05)$ were observed in the caecum as analysed by two-way ANOVA. 


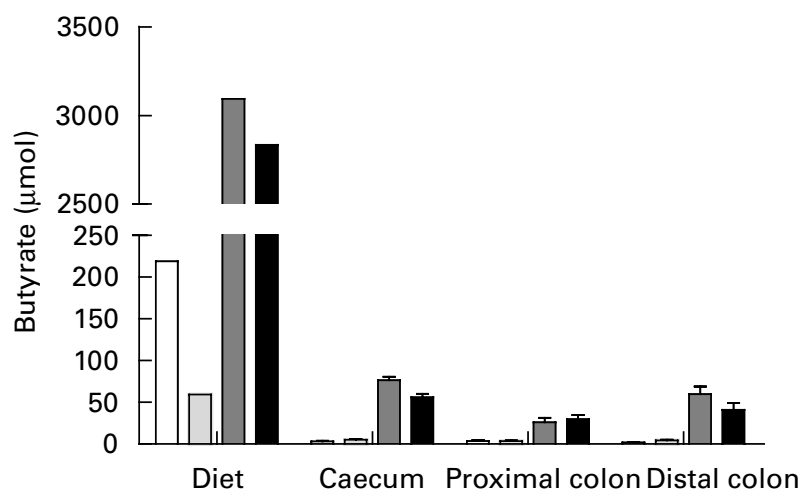

Fig. 7. Butyrate pools released from the digesta solid phase by alkaline hydrolysis from the diet (diet for $24 \mathrm{~h}$ period), caecum (n 7-8), proximal colon $(n$ 3-7) and distal colon ( $n$ 7-8) from rats fed high-amylose maize starch (HAMS; $\square$ ), 'cooked' HAMS ( $\square$ ), butyrylated HAMS (HAMSB; $\square$ ) and 'cooked' HAMSB (ם). Data are means with their standard errors represented by vertical bars.

Portal vein plasma butyrate concentrations were significantly greater in rats fed HAMSB than in those fed HAMS $(P<0.05)$ (Fig. 8); however, there was no significant effect of cooking on the plasma butyrate concentrations of either the HAMS or the HAMSB groups. No significant differences were observed in acetate or propionate concentrations among any of the diets or due to cooking.

\section{Discussion}

The present studies confirm that uncooked unmodified HAMS and HAMSB resist small-intestinal digestion in vitro and raise large-bowel digesta mass and SCFA compared with published values on standard maize starch (Morita et al. 2005). In the case of unmodified HAMS the increase was in total and individual SCFA compared with published standard maize starch (Morita et al. 2005) but with HAMSB there was an additional increase in the acid which had been esterified (Annison et al. 2003; Morita et al. 2005). To our knowledge, the effects of

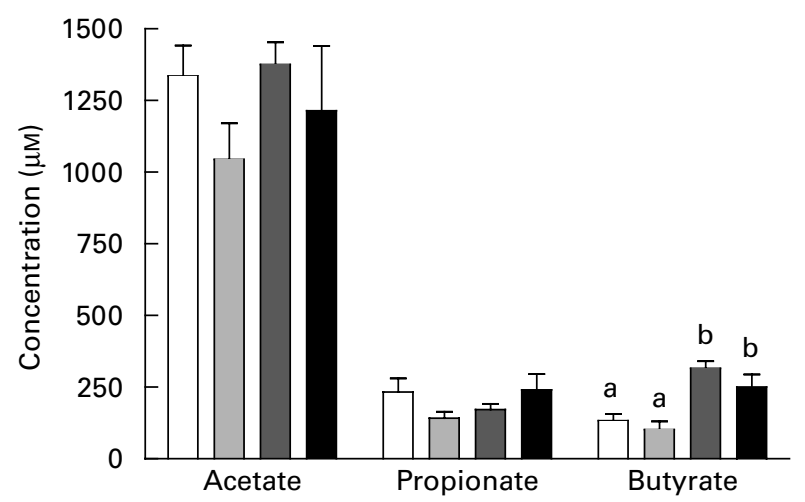

Fig. 8. Acetate, propionate and butyrate concentrations in portal vein plasma samples from rats fed high-amylose maize starch (HAMS; $\square$ ), 'cooked' HAMS ( $\square$ ), butyrylated HAMS (HAMSB; $\square$ ) and 'cooked' HAMSB (ם). Data are means $(n 8)$ with their standard errors represented by vertical bars (except HAMS; $n$ 7). ${ }^{a, b}$ Mean values with unlike letters were significantly different by one-way ANOVA $(P<0.05)$. Analysis by two-way ANOVA showed significant effect of starch type on portal butyrate concentrations $(P<0.0001)$ cooking on the performance of butyrylated starch have not been reported previously. As expected, in vitro amylolysis showed that uncooked HAMSB was extremely resistant to digestion. Cooking increased the amount of glucose released, which is consistent with greater gelatinisation, but approximately $57 \%$ of the starch remained undigested in vitro. Raw HAMS also resisted in vitro amylolysis but cooking decreased the starch remaining to less than $25 \%$ of the total glucosyl units available. Differences in the rate of amylolysis between acylated and unmodified starches have been observed over time periods of 0 to $30 \mathrm{~min}$ (Annison et al. 1995) and between raw and cooked starches over 2 to $6 \mathrm{~h}$ (data not shown). The in vitro amylolysis data obtained for the raw starches are comparable with the percentage starch digestibility observed in colectomised rats of HAMS and acetylated HAMS (Morita et al. 2005). The reduction of in vitro resistance to amylolysis of cooked HAMS by approximately $50 \%$ is consistent with reported in vivo results by ${ }^{13} \mathrm{C}$ breath test analysis in human subjects (Symonds et al. 2004). These authors showed a marked shift from large-bowel production of ${ }^{13} \mathrm{CO}_{2}$ in human subjects consuming uncooked HAMS to small-intestinal digestion when they ate HAMS as pancakes.

The significant decrease in the caecal digesta mass of rats fed HAMS following cooking is consistent with the increase in amylolysis observed in vitro. Although the caecal digesta weight of rats fed HAMSB was not significantly different compared with rats fed HAMS, the disproportionate increase in the butyrate pool is consistent with the bioavailability of the esterified SCFA and accords with previous studies with acylated starches (Annison et al. 2003; Morita et al. 2005). This demonstrates the ability of acylated starches to deliver specific acids to the large bowel in significantly greater amounts than the HAMS

The majority of the free SCFA are found in the caecum as it is the main site of bacterial fermentation of carbohydrates in the rat. In the proximal and distal colon, the butyrate pools of the rats fed HAMSB (both raw and cooked) were not significantly different from the HAMS-fed rats despite the presence of greater amounts of butyrate still esterified to the starch (see Fig. 7). This suggests that either there is limited bacterial activity in the proximal and distal colon of rats, or that colonic uptake of butyrate is exceeding enzymic release and bacterial production. The fermentative capacity is unlikely to be an influence, as a previous study has shown that relevant bacterial populations were not significantly altered in rats fed RS throughout the large bowel over a similar experimental period (Le Blay et al. 2003).

The presence of greater amounts of acetate and propionate in the distal colon of rats fed both raw and cooked HAMS may be attributed to the butyrate remaining esterified to the starch molecules of HAMSB impeding fermentation in the proximal and distal colon. It is likely that the dynamics of HAMSB breakdown in the human large bowel would be different from those observed. Despite the reduction in resistance of cooked HAMS in vitro, there was no significant reduction of SCFA production in vivo following the cooking.

A major benefit of $\mathrm{RS}$ is the maintenance of low $\mathrm{pH}$ throughout the large bowel, which is beneficial in preventing overgrowth of $\mathrm{pH}$-sensitive pathogenic bacteria (Topping \& Clifton, 2001), and preventing the degradation of primary bile acids to carcinogens (Christl et al. 1997; Dongowski 
et al. 2002). In the present study, cooking HAMS significantly reduced the ability of the starch to lower caecal and proximal colonic $\mathrm{pH}$, which may reduce the uptake and use of SCFA produced in the lumen by colonocytes (Ritzhaupt et al. 1998) and enter the circulation. HAMSB that had been cooked could maintain acidic $\mathrm{pH}$ throughout the large bowel. The maintenance of acidic $\mathrm{pH}$ in the distal colon despite no differences in SCFA content may result from decreased digesta ammonia which is known to occur when RS intake is increased (Birkett et al. 1996). However, the reason for the significantly higher $\mathrm{pH}$ for cooked HAMS in the proximal colon is unclear although may be due to a lower amount of RS remaining in the digesta.

A previous study demonstrated survival of acylated starches through the small intestine of colectomised rats (Morita et al. 2005). Approximately 90-95\% of the esterified butyrate was liberated by the time the digesta had left the caecum. The present study demonstrates that starch esterified with butyrate can significantly increase luminal free butyrate by amounts far in excess of those produced HAMS.

Hydrolysis of diet samples and daily food intakes allows an estimation of the amount of esterified butyrate consumed over the last $24 \mathrm{~h}$ period of the experiment. Using $10 \mathrm{ml} / \mathrm{min}$ as an estimate of the average $24 \mathrm{~h}$ portal vein flow in the rat (including sleep and postprandial flow rates) (Mansbach et al. 1991; Mansbach \& Dowell, 1993), we suggest that the majority of the butyrate liberated from the HAMSB was absorbed through the gut wall. This is supported by the suggestion that approximately $95 \%$ SCFA produced in the large bowel are absorbed through the epithelium (Topping \& Clifton, 2001).

Caecal digesta SCFA, particularly butyrate, remain low in rats fed raw or cooked HAMS compared with HAMSB following $10 \mathrm{~d}$ feeding at $10 \%$ of the diet. This may be a result of insufficient time for bacterial adaptation to the high-amylose starch substrate (Henningsson et al. 2003). In contrast, both HAMSB diets generated significantly more butyrate in the same experimental period, demonstrating that esterification of butyrate to starch provides a rapid delivery system for specific SCFA that do not require a period of bacterial adaptation. Once the esterified SCFA have been released, the starch molecule is available for fermentation similar to native RS such as HAMS, which will further increase luminal SCFA.

In conclusion, the present study has demonstrated that butyrylated starch is less susceptible to small-intestinal amylolysis than HAMS in vitro. Cooked butyrylated starches deliver significantly greater amounts of esterified butyrate than raw or cooked HAMS, which are available for the large bowel of rats. The present study also demonstrates that cooking high-amylose starches such as HAMS significantly increases susceptibility to small-intestinal amylolysis in vitro. However, this did not translate to a significant reduction in SCFA pools in the large bowel of the rat although the luminal $\mathrm{pH}$ was significantly higher following cooking, which may increase the risk of luminal exposure to carcinogens. Preparation of carbohydrates to simulate human food for use in animal experiments may be important to assess nutritional and physiological effects accurately. These results suggest the butyrylated starches are an effective delivery system for specific SCFA to the large bowel and have potential to aid in the maintenance of large-bowel heath.

\section{Acknowledgements}

The authors wish to thank National Starch Food Innovation and Ian Brown for providing the HAMS and HAMSB. In addition we would like to thank Dr Tony Bird for his valuable contribution to the study, Julie Dallimore and Brad Klingner for help during the animal study, and Dr Ian Saunders for statistical advice. Balazs Bajka wishes to thank Associate Professor Michael Roberts for his supervision and support.

\section{References}

Ahmad MS, Krishnan S, Ramakrishna BS, Mathan M, Pulimood AB \& Murthy SN (2000) Butyrate and glucose metabolism by colonocytes in experimental colitis in mice. Gut 46, 493-499.

Ahmed R, Segal I \& Hassan H (2000) Fermentation of dietary starch in humans. Am J Gastroenterol 95, 1017-1020.

Andoh A, Tsujikawa T \& Fujiyama Y (2003) Role of dietary fiber and short-chain fatty acids in the colon. Curr Pharm Des 9, 347-358.

Annison G, Illman RJ \& Topping DL (2003) Acetylated, propionylated or butyrylated starches raise large bowel short-chain fatty acids preferentially when fed to rats. J Nutr 133, 3523-3528.

Annison G, Topping DL \& Illman RJ (1995) Fatty acid delivery system comprising a hydrolyzable bond. Commonwealth Scientific and Industrial Research Organization, Australia, patent no. WO9513801 http://ep.espacenet.com.

Asp N-G (1992) Resistant starch - Proceedings from the second plenary meeting of EURESTA: European FLAIR Concerted Action, 11 on physiological implications of the consumption of resistant starch in man. Preface. Eur J Clin Nutr 46, S1.

Birkett A, Muir J, Phillips J, Jones G \& O’Dea K (1996) Resistant starch lowers fecal concentrations of ammonia and phenols in humans. Am J Clin Nutr 63, 766-772.

Brown IL, McNaught KJ \& Moloney E (1995) Hi-Maize(Tm) - new directions in starch technology and nutrition. Food Aust 47, 272-275.

Brown MA, Storlien LH, Brown IL \& Higgins JA (2003) Cooking attenuates the ability of high-amylose meals to reduce plasma insulin concentrations in rats. Br J Nutr 90, 823-827.

Champ M, Langkilde A-M, Brouns F, Kettlitz B \& Le Bail-Collet Y (2003) Advances in dietary fibre characterisation. 2. Consumption, chemistry, physiology and measurement of resistant starch; implications for health and food labelling. Nutr Res Rev 16, 143-161.

Christl SU, Bartram HP, Paul A, Kelber E, Scheppach W \& Kasper H (1997) Bile acid metabolism by colonic bacteria in continuous culture: effects of starch and pH. Ann Nutr Metab 41, 45-51.

Dehghan-Kooshkghazi M \& Mathers JC (2004) Starch digestion, large-bowel fermentation and intestinal mucosal cell proliferation in rats treated with the $\alpha$-glucosidase inhibitor acarbose. $\mathrm{Br} J$ Nutr 91, 357-365.

Dongowski G, Huth M, Gebhardt E \& Flamme W (2002) Dietary fiber-rich barley products beneficially affect the intestinal tract of rats. J Nutr 132, 3704-3714.

Henningsson AM, Margareta E, Nyman GL \& Bjorck IM (2003) Influences of dietary adaptation and source of resistant starch on shortchain fatty acids in the hindgut of rats. Br J Nutr 89, 319-328.

Inatomi $\mathrm{O}$, Andoh $\mathrm{A}$, Kitamura $\mathrm{K}$, Yasui $\mathrm{H}$, Zhang $\mathrm{Z} \&$ Fujiyama $\mathrm{Y}$ (2005) Butyrate blocks interferon-gamma-inducible protein-10 release in human intestinal subepithelial myofibroblasts. $J$ Gastroenterol 40, 483-489.

Kleessen B, Stoof G, Proll J, Schmiedl D, Noack J \& Blaut M (1997) Feeding resistant starch affects fecal and cecal microflora and short-chain fatty acids in rats. J Anim Sci 75, 2453-2462.

Le Blay GM, Michel CD, Blottiere HM \& Cherbut CJ (2003) Raw potato starch and short-chain fructo-oligosaccharides affect the composition and metabolic activity of rat intestinal microbiota 
differently depending on the caecocolonic segment involved. $J$ Appl Microbiol 94, 312-320.

Lee YT \& Chang HG (2004) The effect of heat treatments on in vitro starch digestibility and resistant starch of selected cereals. Food Sci Biotechnol 13, 810-813.

Mansbach CM II \& Dowell RF (1993) Portal transport of long acyl chain lipids: effect of phosphatidylcholine and low infusion rates. Am J Physiol 264, G1082-G1089.

Mansbach CM II, Dowell RF \& Pritchett D (1991) Portal transport of absorbed lipids in rats. Am J Physiol 261, G530-G538.

Morita T, Kasaoka S, Kiriyama S, Brown IL \& Topping DL (2005) Comparative effects of acetylated and unmodified high-amylose maize starch in rats. Starch 57, 246-253.

Murase M, Kimura Y \& Nagata Y (1995) Determination of portal short-chain fatty acids in rats fed various dietary fibers by capillary gas chromatography. J Chromatogr 664B, 415-420.

National Health and Medical Research Council (2004) Australian code of practice for the care and use of animals for scientific purposes, 7th ed. Accessed 29 April 2006. http://www.nhmrc.gov.au/ publications/synopses/ea16syn.htm

Ozcan S \& Jackson DS (2005) Functionality behavior of raw and extruded corn maize starch mixtures. Cereal Chem 82, $223-227$.

Pizzoferrato L, Rotilio G \& Paci M (1999) Modification of structure and digestibility of chestnut starch upon cooking: a solid state (13)C CP MAS NMR and enzymatic degradation study. J Agric Food Chem 47, 4060-4063.
Reeves PG, Nielsen FH \& Fahey GC Jr (1993) AIN-93 purified diets for laboratory rodents: final report of the American Institute of Nutrition ad hoc writing committee on the reformulation of the AIN-76A rodent diet. $J$ Nutr 123, 1939-1951.

Ritzhaupt A, Ellis A, Hosie KB \& Shirazi-Beechey SP (1998) The characterization of butyrate transport across pig and human colonic luminal membrane. J Physiol 507, 819-830.

Rondeau MP, Meltzer K, Michel KE, McManus CM \& Washabau RJ (2003) Short chain fatty acids stimulate feline colonic smooth muscle contraction. J Feline Med Surg 5, 167-173.

Scheppach W \& Weiler F (2004) The butyrate story: old wine in new bottles? Curr Opin Clin Nutr Metab Care 7, 563-567.

Symonds EL, Kritas S, Omari TI \& Butler RN (2004) A combined $13 \mathrm{CO} 2 / \mathrm{H} 2$ breath test can be used to assess starch digestion and fermentation in humans. $J$ Nutr 134, 1193-1196.

Topping DL \& Clifton PM (2001) Short-chain fatty acids and human colonic function: roles of resistant starch and nonstarch polysaccharides. Physiol Rev 81, 1031-1064.

Topping DL, Fukushima M \& Bird AR (2003) Resistant starch as a prebiotic and synbiotic: state of the art. Proc Nutr Soc 62, 171-176.

Topping DL, Gooden JM, Brown IL, Biebrick DA, McGrath L, Trimble RP, Choct M \& Illman RJ (1997) A high amylose (amylomaize) starch raises proximal large bowel starch and increases colon length in pigs. J Nutr 127, 615-622.

Young GP, Hu Y, Le Leu RK \& Nyskohus L (2005) Dietary fibre and colorectal cancer: a model for environment-gene interactions. Mol Nutr Food Res 49, 571-584. 